\title{
Methylphenidate Significantly Reduces Lapses of Attention During On-Road Highway Driving in Patients With ADHD
}

\author{
Joris C. Verster, PhD*† and Thomas Roth, PhD
}

\begin{abstract}
Lapses of attention are characteristic for attention-deficit/ hyperactivity disorder (ADHD) and as such may impair performance of daily activities. Data from an on-road driving study were reanalyzed to determine lapses in patients with ADHD after treatment with methylphenidate and placebo.

A total of 18 adult ADHD patients performed a 100-km on-road driving test and were instructed to drive with a steady lateral position and constant speed. The SD of lateral position (SDLP), that is, the weaving of the car, lapses, and alertness, was assessed.

Driving was significantly better $(P=0.006)$ with methylphenidate (SDLP, $18.8 \mathrm{~cm}$ ) when compared with placebo (SDLP, $21.2 \mathrm{~cm}$ ). Both the reduction in SDLP and the number of lapses $(P=0.003)$ confirm this significant improvement, which is further supported by subjective assessments of perceived driving performance. Although lapses were common in the placebo condition (11/18 patients), they were much less frequently observed (5/18 patients) after treatment with methylphenidate. Postdriving assessments suggest that lapses often go unnoticed by drivers.

In conclusion, methylphenidate significantly improves driving of patients with ADHD by significantly reducing the number of lapses.
\end{abstract}

Key Words: ADHD, methylphenidate, driving, lapses, SDLP

(J Clin Psychopharmacol 2014;34: 633-636)

Ed ach year, many children and adults are diagnosed with attention- deficit/hyperactivity disorder (ADHD). Because hyperactive behavior often diminishes when growing older, it has long been believed that ADHD disappears after adolescence. However, current evidence shows that ADHD and attention deficits often persist in adulthood. ${ }^{1}$ These are reflected in daily activities such as academic achievement, work performance, and potentially dangerous activities such as driving a car.

Methylphenidate is the most common pharmacological treatment of ADHD and is regarded as the first-line treatment. ${ }^{2}$ An important clinical improvement seen after pharmacological treatment is the positive effects on sustained attention. Studies have shown that 1 manifestation of this reduction in distraction is safer driving. Untreated ADHD has been linked to an increased risk of speeding and a 4-fold increased risk of having an accident. ${ }^{3,4}$ When treated effectively with methylphenidate, various aspects of driving performance were shown to improve significantly. ${ }^{4}$

From the *Division of Pharmacology, Utrecht Institute for Pharmaceutica Sciences, Utrecht University, Utrecht, the Netherlands; †Centre for Human Psychopharmacology, Swinburne University, Melbourne, Victoria, Australia; and \$Sleep Disorders and Research Center, Henry Ford Health System, Detroit, MI.

Received July 5, 2013; accepted after revision April 7, 2014.

This study was funded by Utrecht University.

Reprints: Joris C. Verster, PhD, Division of Pharmacology, Utrecht Institute for Pharmaceutical Sciences, Utrecht University, Universiteitsweg 99,

3584 CG, Utrecht, the Netherlands (e-mail: j.c.verster@uu.nl).

Copyright (C) 2014 by Lippincott Williams \& Wilkins

ISSN: 0271-0749

DOI: $10.1097 / \mathrm{JCP} .0000000000000174$
Driver inattention has been found as the most important contributing factor in rear-end collisions ${ }^{5}$ and is a common feature of impaired driving, especially in sleepiness-related accidents. ${ }^{6}$ Driving performance deteriorates during episodes of inattention. ${ }^{7}$ An American study shows that driving while drowsy was a contributing factor for $22 \%$ to $24 \%$ of the crashes and near-crashes. ${ }^{8}$ Lapses of attention may be a cause or predictor of traffic accidents. ${ }^{9}$ Lapses of attention are common in individuals with untreated ADHD because inattention is the primary feature of this medical condition. ${ }^{10}$ They have been described as periods of "microsleep," which are commonly observed in patients with ADHD. ${ }^{11}$ In 2008, it was shown that effective treatment with methylphenidate significantly reduced the SD of lateral position (SDLP), and thus improved driving performance of adult ADHD patients. ${ }^{12}$ The current reanalysis of these data aims to measure lapses directly and compare them with the SDLP and the outcomes of subjective driving quality and alertness.

\section{METHODS}

The study was conducted as a double-blind, placebocontrolled, randomized, crossover design. Patients were included if they used a stable daily dose of methylphenidate, did not experience adverse effects from the treatment, and were considered to be a clinical responder by the treating physician. A detailed description of the diagnosis of patients and study methodology can be read elsewhere. ${ }^{12}$

Treatments (immediate release methylphenidate [patients' regular dosage] or placebo) were administered orally with $240 \mathrm{~mL}$ tap water, 1.5 hours before performing the on-the-road driving test. If applicable, a second treatment dose was administered according to the patient's usual interval between the first and second dose. A standardized driving test was performed on a 2-lane primary highway during normal traffic on a 100-km track between the cities of Utrecht and Arnhem. ${ }^{13}$ Patients were instructed to drive with a steady lateral position within the right (slower) traffic lane while maintaining a constant speed of $95 \mathrm{~km} / \mathrm{h}$. Patients were allowed to deviate from the instructions to overtake a slower moving vehicle in the same traffic lane. A licensed driving instructor who had access to dual controls sat in the right front seat, guarding the patient's safety during the driving test. Driving tests could be terminated before completion if the driving instructor or the patient felt it was unsafe to continue.

The SDLP $(\mathrm{cm})$, that is, the amount of weaving of the car, and the SD of speed $(\mathrm{km} / \mathrm{h})$ were computed from the continuous lateral position and speed measurements. ${ }^{13}$ In addition, raw driving test data were examined to determine if lapses were present. ${ }^{9}$ A lapse was defined as a change in lateral position of greater than $100 \mathrm{~cm}$, lasting for at least 8 seconds. ${ }^{9}$ The data were analyzed for (1) the number of lapses and (2) the duration of each lapse. From these data, 2 outcome measures were computed as follows: (1) \#lapses

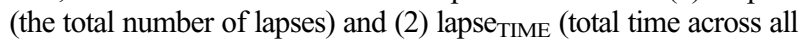
lapses together).

Subjective assessments of driving quality and mental effort to perform the test were made directly after the driving test. ${ }^{13}$ Patients rated 6 dimensions of their driving style on a 10-cm bipolar visual analog scale (VAS), including foolish-wise, 
TABLE 1. Summary of the Study Results

\begin{tabular}{lccc}
\hline & Methylphenidate & Placebo & $\boldsymbol{P}$ \\
\hline Number of lapses & $0.4(1.0)$ & $2.4(3.1)$ & 0.003 \\
Total lapse time, s & $8.1(18.0)$ & $35.9(47.6)$ & 0.017 \\
SDLP, cm & $18.8(3.5)$ & $21.2(4.0)$ & 0.006 \\
Subjective driving quality & $13.1(4.2)$ & $9.9(5.1)$ & 0.026 \\
Mental effort & $4.6(2.2)$ & $6.5(2.6)$ & 0.030 \\
Unpredictable-predictable & $8.2(1.2)$ & $7.1(2.2)$ & 0.016 \\
Dangerous-safe & $7.9(1.6)$ & $6.8(2.4)$ & 0.051 \\
Tensed-relaxed & $7.0(1.8)$ & $5.0(2.6)$ & 0.008 \\
Foolish-wise & $8.0(1.6)$ & $6.5(2.4)$ & 0.043 \\
Inconsiderate-considerate & $7.9(1.6)$ & $6.9(2.1)$ & 0.107 \\
Irresponsible-responsible & $8.2(1.7)$ & $7.0(2.4)$ & 0.080 \\
\hline
\end{tabular}

The mean (SD) of 18 patients are shown. Differences are significant if $P<0.05$.

inconsiderate-considerate, dangerous-safe, tense-relaxed, unpredictablepredictable, and irresponsible-responsible. ${ }^{14}$ Subjects rated their sleepiness on a 10-cm scale ranging from sleepy to alert, and mood was assessed with the Addiction Research Center Inventory 49 (ARCI-49) ${ }^{15}$ and the Profile of Mood States-Short Form (POMSSF) ${ }^{16}$ The ARCI- 49 comprises 49 yes/no questions that relate to 5 factors differentiating between mood changes induced by psychoactive drugs. The 5 factors assessed were (1) euphoria (morphine group), (2) dysphoria (lysergic acid diethylamide group), (3) sedation (pentobarbital-chlorpromazine-alcohol group), (4) intellectual efficacy and energy (benzedrine group), and (5) activation (amphetamine group). The POMS-SF contains 37 items and comprises 5 subscales, including (1) tension, (2) depression, (3) anger, (4) fatigue, and (5) vigor. Patients were asked to respond to each item on a 5-point Likert scale, from 0 (not at all) to 4 (extremely), to express how they were feeling at that moment. Assessments were completed at baseline ( $\mathrm{T} 0$, just before treatment), before the driving test (T60, 60 minutes after intake), after the driving test (T160, 160 minutes after intake), and at the end of the test day (T200, 200 minutes after intake).

Statistical analyses were conducted using paired $t$ tests to compare placebo and methylphenidate condition. In case data were not normally distributed, Friedman 2-way analysis of variance ranks was applied to compare the 2 conditions. Difference scores (placebo-methylphenidate) were computed and correlated with each other. Pearson $r$ correlations were computed in case of a normal data distribution; otherwise, Spearman correlations were computed. Results were considered significant if $P<0.05$ (2-sided).

\section{RESULTS}

Eighteen patients diagnosed with ADHD (combined type) participated in the study. The driving test data are summarized in Table 1.

From Table 1, it is evident that methylphenidate significantly improves driving ability in patients with ADHD. Both a reduction in $\operatorname{SDLP}(P=0.006)$ and the number of lapses $(P=0.003)$ confirm this significant improvement, which is further supported by subjective assessments of perceived driving performance. Although lapses are common in the placebo condition (11 of 18 patients), they are significantly less frequently observed ( 5 of 18 patients) after treatment with methylphenidate. In line, lapse TIME $_{\text {was significantly }}$ reduced after treatment with methylphenidate. There was a significant correlation of $\Delta$ SDLP with the $\Delta$ \#lapses $(r=0.626, P=0.005)$ and the $\Delta$ lapse $\mathrm{TIME}_{\mathrm{TIM}}(r=0.618, P=0.006)$. The SDLP and the distribution of lapses over distance traveled are shown in Figure 1.

From Figure 1, vigilance decrement is evident in that over distance traveled SDLP increases. Likewise, the number of lapses progressively increases over distance traveled, and they most frequently occur in the second $50-\mathrm{km}$ part of the driving test.

Subjective assessments revealed that after methylphenidate, driving quality was significantly improved $(P=0.023)$, and mental effort to perform the test was significantly reduced $(P=0.022)$. After methylphenidate, patients reported that their driving style was significantly less unpredictable $(P=0.004)$, less dangerous $(P=$ $0.043)$, less foolish $(P=0.034)$, and less tensed $(P=0.005)$. Difference scores on many of these driving style items correlated significantly with $\Delta$ SDLP, whereas none of them correlated significantly with $\Delta$ \#llapses and $\Delta$ lapse $_{\text {TIME }}($ Table 2). This suggests that lapses may go unnoticed, except when large deviations from the average lateral position are detected (and likely to be viewed as unsafe and reflecting poorer driving quality).

Most subscales of the POMS-SF and ARCI-49 showed no significant differences on the mood subscales between methylphenidate and placebo. As expected, significant treatment effects were found for POMS-SF subscales on vigor $(P=0.034)$ and fatigue $(P=$ $0.045)$. However, placebo-methylphenidate differences on the ARCI-49 sedation subscale did not reach significance $(P=0.068)$ nor did the scores on the VAS scale measuring sleepiness $(P=$ 0.102 ). Methylphenidate and placebo scores on these alertnessrelated scales are summarized in Figure 2.

Although not all methylphenidate-placebo differences on these subscales reached significance, the overall picture is that in the placebo condition, alertness is reduced after completing
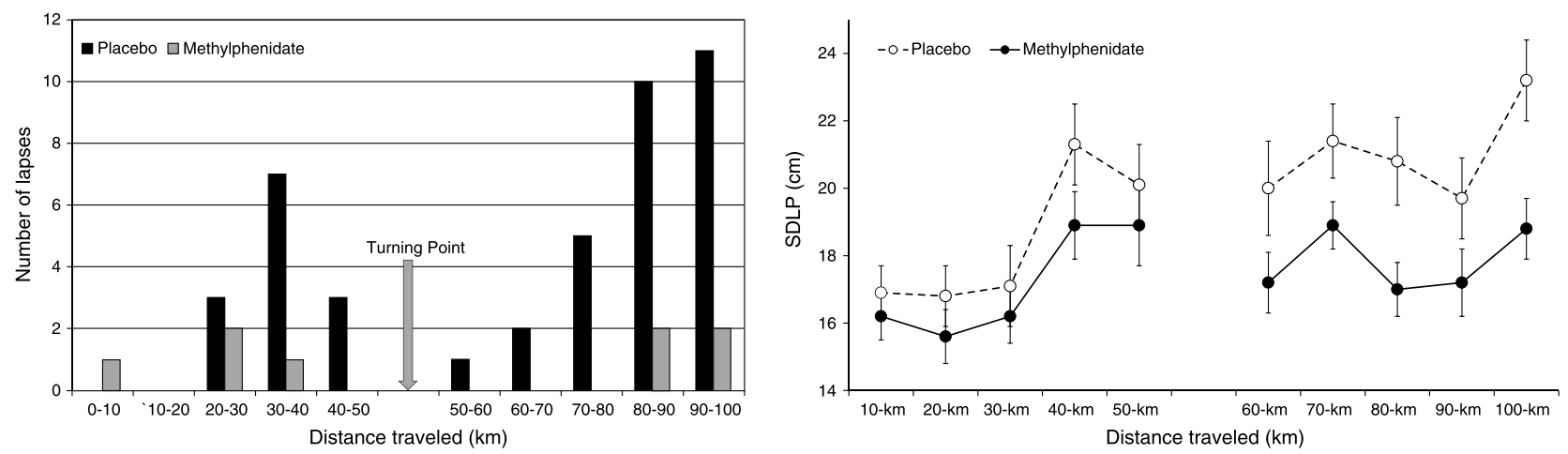

FIGURE 1. SDLP and number of lapses over distance traveled. Methylphenidate significantly improves driving by reducing both the number of lapses (left) and SDLP (right). 
TABLE 2. Correlations and Their Significance

\begin{tabular}{lccc}
\hline & $\Delta$ SDLP & $\Delta$ \#Lapses & $\Delta$ Lapse $_{\text {TIME }}$ \\
\hline$\Delta$ SDLP & - & & \\
$\Delta$ \#lapses & $0.626^{*}$ & - & \\
$\Delta$ Lapse $_{\text {TIME }}$ & $0.618^{*}$ & $0.952^{*}$ & - \\
$\Delta$ Subjective driving quality $^{*}-0.748^{*}$ & -0.335 & -0.286 \\
$\Delta$ Mental effort & $0.674^{*}$ & 0.336 & 0.323 \\
$\Delta$ Unpredictable-predictable & -0.340 & -0.053 & -0.061 \\
$\Delta$ Dangerous-safe & $-0.615^{*}$ & -0.232 & -0.217 \\
$\Delta$ Tensed-relaxed & $-0.648^{*}$ & -0.361 & -0.322 \\
$\Delta$ Unwise-wise & -0.427 & -0.135 & 0.078 \\
$\Delta$ Irrational-rational & $-0.497^{* *}$ & -0.284 & -0.047 \\
$\Delta$ Irresponsible-responsible & -0.387 & 0.256 & 0.062 \\
\hline
\end{tabular}

Difference scores ( $\Delta$, placebo-methylphenidate) were used.

* Significant correlations are indicated by $P<0.01$.

**Significant correlations are indicated by $P<0.05$.

the $100-\mathrm{km}$ driving test (T160). The stimulant properties of methylphenidate significantly counteract this effect.

Comparing scores on the alertness-related scales to objective measures of driving performance (ie, SDLP and the occurrence of lapses) confirmed our observations that lapses may go unnoticed by drivers. Difference scores before the driving test (T60) did not correlate significantly with any objective driving test outcome measures. After the driving test (T160), $\Delta$ SDLP correlated significantly with difference scores on VAS sleepiness $(r=0.488, P=$ $0.040)$ and POMS-SF fatigue $(r=0.493, P=0.038)$. In contrast,
$\Delta$ \#lapses scores did not correlate significantly with any subjective assessment. $\Delta$ Lapse $_{\mathrm{TIME}}$ correlated significant with $\triangle \mathrm{POMS}-\mathrm{SF}$ fatigue scores $(r=0.498, P=0.035)$.

\section{DISCUSSION}

This study confirms previous findings that methylphenidate treatment significantly improves driving performance of individuals with ADHD. ${ }^{9}$ The observed SDLP difference was of the same magnitude as seen when driving with a blood alcohol concentration of $0.05 \%$, that is, the legal limit for driving in many countries. ${ }^{17}$ A significant reduction in the number of lapses during highway driving was seen relative to placebo treatment. The importance of these findings lies in the fact that lapses of attention is the core deficit observed in adult ADHD patients. This study shows that effective treatment reduces or prevents the occurrence of the lapses in driving and reduces the total lapse time. As such, it is direct evidence that successful treatment is effective in potentially dangerous activities such as driving a car.

A lapse is typically thought of as a momentary loss of attention. Thus small errors are small (ie, SDLP changes) because the subject remains aware and performs a corrective action. In contrast, lapses are large errors because the subjects are not aware (maybe microsleep), lapses are the result of not taking corrective action. Hence, the lack of awareness is inherent in a lapse. Subjective assessments of driving performance and alertness reveal that these are significantly related with SDLP. In other words, this suggests that patients were aware of increased weaving in placebo conditions and correctly identified their driving as worse when compared with that in methylphenidate-treated conditions. In contrast, the number of lapses and the total lapse time did not correlate with these subjective assessments. This absence of a significant correlation suggests that lapses often seem to go
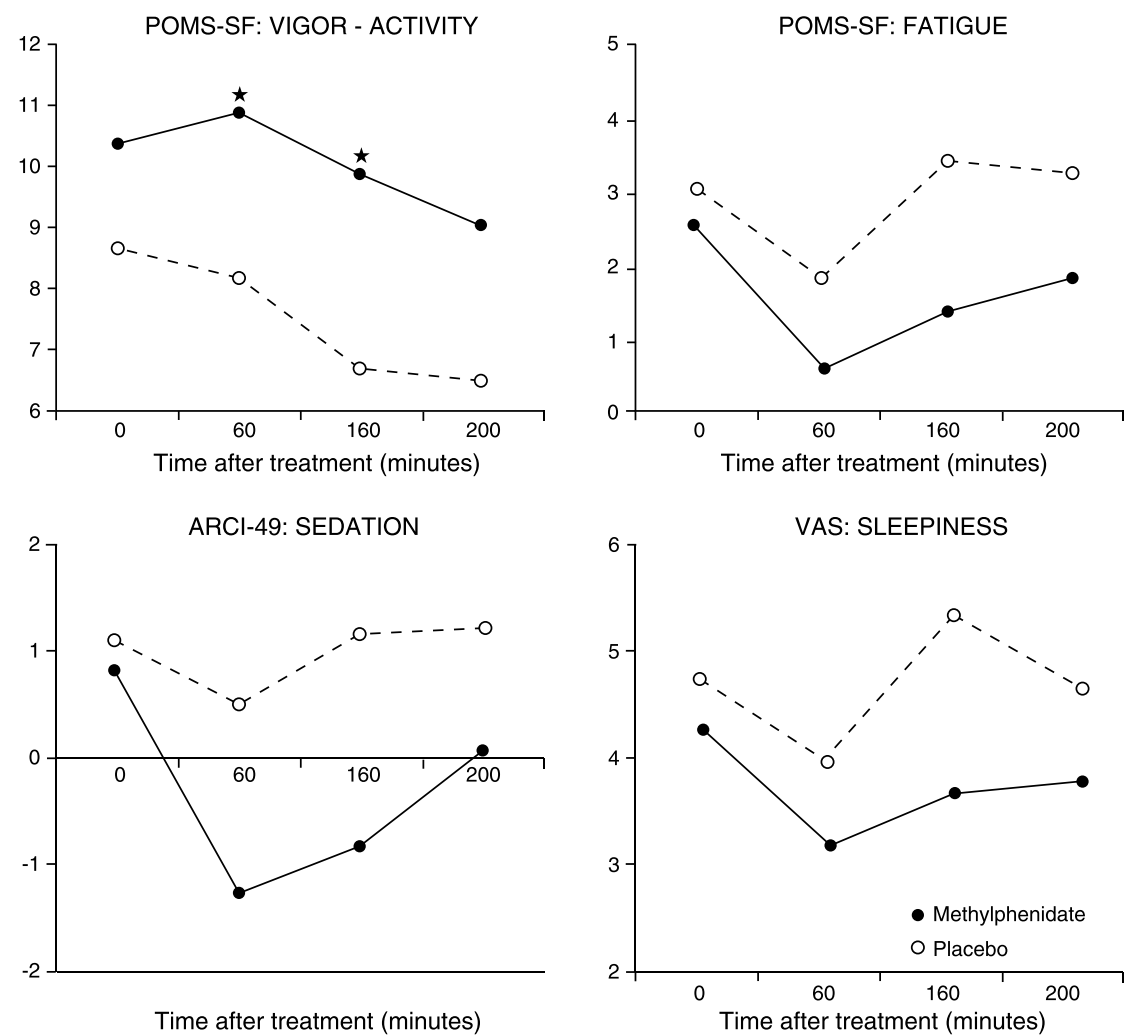

FIGURE 2. Subjective assessments of sleepiness and sedation. Asterisk $\left(^{*}\right)$ indicates the significant differences $(P<0.05)$. 
unnoticed. It is understandable that experiencing a large lapse, which causes the patient to drive the car out of lane, can serve as a wake-up call, making patients aware that their driving is unsafe. When lapses go unnoticed, however, this is worrisome in terms of traffic safety because these patients may perceive their driving as unimpaired. Future research should examine the awareness of lapses in more detail to confirm these hypotheses. Future research should also focus on the relationship between having lapses and the risk of having accidents. In the on-road driving test, accidents do not happen because the driver is accompanied by a driving instructor with dual controls. ${ }^{13}$ In driving simulator research, it has been reported that crashes occur, and sometimes they are even used as the outcome measure of the test. ${ }^{4}$ It would be interesting to reanalyze these data for the occurrence of lapses and to determine how these relate to the chances of having a crash. Taken together, the demonstration of a significant reduction of the number of lapses of attention and the total lapse time during driving confirm that successful treatment with methylphenidate effectively improves the core deficit of adult ADHD.

\section{AUTHOR DISCLOSURE INFORMATION}

The authors have no conflicts of interest to disclose. Dr Verster has received grants/research support from the Dutch Ministry of Infrastructure and the Environment, Red Bull GmbH, and Takeda Pharmaceuticals and has acted as a consultant for the Canadian Beverage Association, Centraal Bureau Drogisterijbedrijven, Coleman Frost, Deenox, INSV, Purdue, Red Bull GmbH, SanofiAventis, Sepracor, Takeda, Transcept, and Trimbos Institute. Dr Roth has received grants/research support from Aventis, Cephalon, GlaxoSmithKline, Neurocrine, Pfizer, Sanofi, ScheringPlough, Sepracor, Somaxon, Syrex, Takeda, TransOral, Wyeth, and XenoPort and has acted as a consultant for Abbott, Acadia, Acoglix, Actelion, Alchemers, Alza, Ancil, Arena, AstraZeneca, Aventis, AVER, BMS, BTG, Cephalon, Cypress, Dove, Elan, Eli Lilly, Evotec, Forest, GlaxoSmithKline, Hypnion, Impax, Intec, Intra-Cellular, Jazz, Johnson \& Johnson, King, Lundbeck, McNeil, MediciNova, Merck, Neurim, Neurocrine, Neurogen, Novartis, Orexo, Organon, Prestwick, Procter \& Gamble, Pfizer, Purdue, Resteva, Roche, Sanofi, Schering-Plough, Sepracor, Servier, Shire, Somaxon, Syrex, Takeda, TransOral, Vanda, VivoMetrics, Wyeth, Yamanuchi, and XenoPort.

\section{REFERENCES}

1. Post RE, Kurlansik SL. Diagnosis and management of adult attention-deficit/hyperactivity disorder. Am Family Physician. 2012; $85: 890-896$

2. Retz W, Retz-Junginger P, Thome J, et al. Pharmacological treatment of adult ADHD in Europe. World J Biol Psychiatry. 2011;12:89-94.
3. Barkley RA, Murphy KR, Dupaul GJ, et al. Driving in young adults with attention deficit hyperactivity disorder: knowledge, performance, adverse outcomes, and the role of executive functioning. J Int Neuropsychol Soc. 2002;8:655-672.

4. Jerome L, Segal A, Habinski L. What we know about ADHD and driving risk: a literature review, meta-analysis and critique. J Can Acad Child Adolesc Psychiatry. 2006;15:105-125.

5. Fort A, Collette B, Bueno M, et al. Impact of totally and partially predictive alert in distracted and undistracted subjects: en event related potential study. Accid Anal Prev. 2013;50:578-586.

6. Blanco M, Biever WJ, Gallagher JP, et al. The impact of secondary task cognitive processing demand on driving performance. Accid Analysis Prev. 2006;38:895-906.

7. Boyle LN, Tippin J, Paul A, et al. Driver performance in the moments during a microsleep. Transp Res Part F Traffic Psychol Behav. 2008;11:126-136.

8. Klauer SG, Dingus TA, Neale VL, et al. The impact of driver inattention on near-crash/crash Risk: an analysis using the 100-car naturalistic driving study data. NHTSA 2006, Report No. DOT HS 810594

9. Verster JC, Bervoets AC, de Klerk S, et al. Lapses of attention as outcome measure of the on-the-road driving test. Psychopharmacology. 2014, 231:283-292.

10. Cox DJ, Madaan V, Cox BS. Adult attention-deficit/hyperactivity disorder and driving: why and how to manage it. Curr Psychiatry Rep. 2011;13: 345-350.

11. Miano S, Parisi P, Villa MP. The sleep phenotypes of attention deficit hyperactivity disorder: the role of arousal during sleep and implications for treatment. Med Hypotheses. 2012;79:147-153.

12. Verster JC, Bekker EM, de Roos M, et al. Methylphenidate significantly improves driving ability of adults with attention-deficit hyperactivity disorder: a randomized crossover trial. J Psychopharmacol. 2008;22: 230-239.

13. Verster JC, Roth T. Standard operation procedures of conducting the on-the-road driving test, and measurement of the standard deviation of latera position (SDLP). Int J Gen Med. 2011;4:359-371.

14. McCormick IA, Walkey FH, Green DE. Comparative perceptions of driver ability — a confirmation and expansion. Accid Anal Prev. 1986;18:205-208.

15. Haertzen CA, Hill HE, Belleville RE. Development of the Addiction Research Center Inventory (ARCI): selection of items that are sensitive to the effects of various drugs. Psychopharmacologia. 1963;4:155-166.

16. Curran SL, Andrykowski MA, Studts JL. Short Form of the Profile of Mood States (POMS-SF): psychometric information. Psychol Assess. $1995 ; 7: 80-83$.

17. Louwerens JW, Gloerich ABM, De Vries G, et al. The relationship between drivers' blood alcohol concentration (BAC) and actual driving performance during high speed travel. In: Noordzij PC, Roszbach R, eds. Alcohol, Drugs and Traffic Safety. Proceedings of the 10th International Conference on Alcohol, Drugs and Traffic Safety. Amsterdam, the Netherlands: Excerpta Medica; 1987:183-192. 